\title{
Early Antibiotic Exposure Is Not Detrimental to Therapeutic Effect from Immunotherapy in Hepatocellular Carcinoma
}

\author{
Petros Fessas $^{a}$ Muntaha Naeem ${ }^{a}$ Matthias Pinter ${ }^{b}$ Thomas U. Marron ${ }^{c}$ David Szafron ${ }^{d}$ \\ Lorenz Balcar $^{b}$ Anwaar Saeed ${ }^{\text {e Tomi Jun }}{ }^{c}$ Sirish Dharmapuric Anuhya Gampa ${ }^{f}$ \\ Yinghong Wang $^{g}$ Uqba Khan ${ }^{\mathrm{h}}$ Mahvish Muzaffar ${ }^{\mathrm{i}}$ Musharraf Navaid ${ }^{\mathrm{i}}$ \\ Pei-Chang Lee ${ }^{\mathrm{j}, \mathrm{k}}$ Anushi Bulumulle ${ }^{\mathrm{i}}$ Bo Yu ${ }^{\mathrm{u}}$ Sonal Paul' Neil Nimkarl Dominik Bettinger ${ }^{\mathrm{m}}$ \\ Hannah Hildebrand ${ }^{\mathrm{e}}$ Yehia I. Abugabal $^{\mathrm{n}}$ Tiziana Pressiani $^{\circ}$ Nicola Personeni ${ }^{\mathrm{o}, \mathrm{p}}$ \\ Naoshi Nishida ${ }^{q}$ Masatoshi Kudo ${ }^{q}$ Ahmed Kaseb ${ }^{n}$ Yi-Hsiang Huang ${ }^{j, k}$ Celina Ang ${ }^{c}$ \\ Anjana Pillai ${ }^{f} \quad$ Lorenza Rimassa $^{o, p} \quad$ Abdul Rafeh Naqash ${ }^{r}$ Elad Sharon ${ }^{s}$ Alessio Cortellini ${ }^{a, t}$
}

David J. Pinatoa

\begin{abstract}
aDepartment of Surgery and Cancer, Imperial College London, Hammersmith Hospital, London, UK; ${ }^{b}$ Division of Gastroenterology and Hepatology, Department of Internal Medicine III, Vienna Liver Cancer Study Group, AKH and Medical University of Vienna, Vienna, Austria; 'Division of Hematology/Oncology, Department of Medicine, Tisch Cancer Institute, Mount Sinai Hospital, New York, NY, USA; dDepartment of Internal Medicine, Baylor College

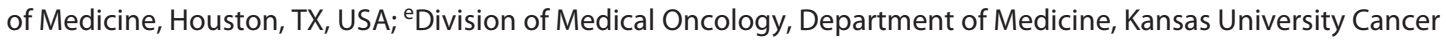
Center, Westwood, KS, USA; fSection of Gastroenterology, Hepatology and Nutrition, The University of Chicago Medicine, Chicago, IL, USA; ${ }^{9}$ Department of Gastroenterology, Hepatology and Nutrition, The University of Texas MD Anderson Cancer Center, Houston, TX, USA; 'hivision of Hematology and Oncology, Weill Cornell Medicine/ New York Presbyterian Hospital, New York, NY, USA; 'Division of Hematology/Oncology, East Carolina University, Greenville, NC, USA; jDivision of Gastroenterology and Hepatology, Department of Medicine, Taipei Veterans General Hospital, Taipei, Taiwan; kInstitute of Clinical Medicine, National Yang Ming Chiao Tung University, Taipei, Taiwan; 'New York Presbyterian Brooklyn Methodist Hospital, New York, NY, USA; mDepartment of Medicine II, Faculty of Medicine, Medical Center University of Freiburg, University of Freiburg, Freiburg, Germany; ${ }^{\text {nDepartment }}$ of Gastrointestinal Medical Oncology, The University of Texas MD Anderson Cancer Center, Houston, TX, USA; o Medical Oncology and Hematology Unit, Humanitas Cancer Center, Humanitas Clinical and Research Center IRCCS, Milan, Italy; ${ }^{\text {DDepartment }}$ of Biomedical Sciences, Humanitas University, Milan, Italy; ${ }^{9}$ Department of Gastroenterology and Hepatology, Kindai University Faculty of Medicine, Osaka, Japan; 'Division of Cancer Treatment and Diagnosis, National Cancer Institute, Bethesda, MD, USA; ${ }^{5}$ National Cancer Institute, Cancer Therapy Evaluation Program, Bethesda, MD, USA; ${ }^{t}$ Department of Biotechnological and Applied Clinical Sciences, University of L'Aquila, L'Aquila, Italy; 'Lincoln Medical Center, New York, NY, USA
\end{abstract}

\section{Keywords}

Antibiotics · Cancer immunotherapy · Immune checkpoint inhibitors · Hepatocellular carcinoma · Gut microbiota

Petros Fessas, Muntaha Naeem, Alessio Cortellini and David J. Pinato contributed equally to the manuscript.

karger@karger.com
www.karger.com/lic

Karger $\stackrel{\text { ' }}{5}$
Published by S. Karger AG, Basel

This article is licensed under the Creative Commons Attribution 4.0 International License (CC BY) (http://www.karger.com/Services/ OpenAccessLicense). Usage, derivative works and distribution are permitted provided that proper credit is given to the author and the original publisher.

\section{Abstract}

Background and Rationale: Immune checkpoint inhibitor (ICI) therapy is an expanding therapeutic option for hepatocellular carcinoma (HCC). Antibiotics (ATB) taken prior to or early during $\mathrm{ICl}$ therapy can impact immunotherapy efficacy across indications; however, the effect of ATB is undefined in HCC. Methods: In a large international cohort of $450 \mathrm{ICl}$ re- 
cipients from Europe, North America, and Asia, we categorized patients according to timing of ATB focusing on exposure within -30 to +30 days from $\mathrm{ICl}$ (early immunotherapy period [EIOP]). ElOP was evaluated in association with overall survival (OS), progression-free survival (PFS), and best radiologic response using RECIST 1.1 criteria. Results: Our study comprised mostly cirrhotic $(329,73.3 \%)$ males $(355,79.1 \%)$ with a Child-Turcotte Pugh class of A $(332,73.9 \%)$, receiving $\mathrm{ICl}$ after 1 therapy line $(251,55.9 \%)$ for HCC of Barcelona clinic liver cancer stage $C(325,72.4 \%)$. EIOP $(n=170,37.9 \%)$ was independent of baseline clinicopathologic features of HCC and correlated with longer PFS (6.1 vs. 3.7 months, log-rank $p=0.0135)$. ElOP + patients had similar OS, overall response, and disease control rates (DCRs) compared to EIOP. The effect of EIOP persisted in landmark time analyses and in multivariable models, confirming the independent predictive role of EIOP in influencing PFS following adjustment for covariates reflective of tumor burden, liver function, and $\mathrm{ICI}$ regimen administered. In patients receiving programmed cell death-1 receptor/ligand inhibitors monotherapy, EIOP was also associated with higher DCRs (61.4\% vs. $50.9 \%, p=$ 0.0494). Conclusions: Unlike other oncological indications, ATB in the 30 days before or after ICl initiation is associated with improved benefit from immunotherapy, independent of disease and treatment-related features. Evaluation of the immune microbiologic determinants of response to $\mathrm{ICl}$ in HCC warrants further investigation.

(c) 2021 This is a work of the U.S. Government and is not subject to copyright protection in the United States. Foreign copyrights may apply. Published by S. Karger AG, Basel.

\section{Introduction}

Despite advancements in diagnosis and management, patients affected by hepatocellular carcinoma (HCC) have a probability of surviving 5 years after the diagnosis of $<15 \%$, making HCC the 4th leading cause of cancer deaths worldwide [1]. Challenges in its management stem from the high proportion of diagnoses that happen incidentally at an advanced stage, when treatment with curative intent is not an available option, resulting in life-limiting prognosis [2]. Cytotoxic chemotherapy has no role in the management of HCC and although sequential use of molecularly targeted therapies improves survival in advanced disease, therapeutic resistance limits long-term survivorship [3].

The survival plateau from locoregional and systemic therapies has stimulated the development of novel pharmacological strategies for the treatment of HCC and in particular the rise to prominence of immune checkpoint inhibitors (ICIs). Monoclonal antibodies against programmed cell death-1 receptor/ligand (PD1/PD-L1) and the cytotoxic T-cell lymphocyte-associated antigen- 4 are capable of inducing tumor immune rejection [4]. However, inhibition of neither of these targets alone is sufficient to translate into significant survival benefit in patients with HCC [5], a finding that has paved the way for the development of immunotherapy combinations such as atezolizumab and bevacizumab, the only regimen associated with an improvement in patient's survival compared to standard of care sorafenib [6].

In view of the immune-mediated mechanism of action of ICI, considerable interest has been devoted to the investigation of how concomitant therapies with a potential immune modulatory effect might interact with the pharmacodynamic properties of ICI, potentially modifying efficacy or enhancing toxicity in cancer patients [7]. Antibiotics (ATB) are known to exert profound immunomodulatory effects, primarily through perturbation of the gut microbiota [8]. Broad-spectrum ATB induce a selective pressure within the gut ecosystem that can reduce bacterial diversity and provide a competitive edge for species that negatively affect response to ICI [9]. ATB-induced gut dysbiosis and loss of microbial diversity have been shown to alter ICI efficacy and may increase the risk of toxicity to ICI [10]. A growing number of clinical studies have reproducibly documented the detrimental effect on objective response, progression-free survival (PFS), and overall survival (OS) from exposure to ATB either prior to or early during the course of ICI therapy, suggesting that ATB might have a preconditioning effect on cancer-specific immune control [11].

While studies have been conducted to evaluate the impact of ATB alongside ICI in other malignancies [12], clinical data documenting the relationship between ATB and outcome from immunotherapy are lacking in HCC, a disease in which the gut-liver axis is profoundly implicated in the pathogenesis and progression of chronic liver disease and cancer [13]. This study was designed to assess whether exposure and timing of ATB treatment is associated with radiologic response and survival in HCC patients treated with ICI.

\section{Methods}

Study Population

From an international consortium of 12 tertiary care referral centers located in Europe, the USA, and Asia, we accrued a prospectively maintained cohort of HCC patients undergoing treat- 
Table 1. Clinical characteristics of the studied patient cohorts

\begin{tabular}{|c|c|c|c|c|}
\hline Median (range) & $65(15-89)$ & $65(18-89)$ & $65(15-87)$ & \multirow{2}{*}{$p=0.8333$} \\
\hline$\geq 70$ years & $119(26.5)$ & $74(26.5)$ & $45(26.5)$ & \\
\hline \multicolumn{5}{|l|}{ Gender } \\
\hline \multicolumn{5}{|l|}{ ECOG-PS } \\
\hline 0 & $178(39.6)$ & $110(39.4)$ & $68(40.0)$ & \multirow{4}{*}{$p=0.2230^{*}$} \\
\hline 1 & $177(39.4)$ & $115(41.2)$ & $115(41.2)$ & \\
\hline 2 & $18(4.0)$ & $8(2.9)$ & $8(2.9)$ & \\
\hline Unknown & $76(16.9)$ & $46(16.5)$ & $30(17.6)$ & \\
\hline \multicolumn{5}{|l|}{ Cirrhosis } \\
\hline HBV and/or HCV infection & $271(60.4)$ & $160(57.3)$ & $111(65.3)$ & $p=0.0954$ \\
\hline \multicolumn{5}{|l|}{ CTP class } \\
\hline A & $332(73.9)$ & 209 (74.) & $123(72.4)$ & \multirow{2}{*}{$p=0.5697$} \\
\hline B & $117(26.1)$ & $70(25.1)$ & $47(27.6)$ & \\
\hline \multicolumn{5}{|l|}{ BCLC stage } \\
\hline$A$ & $15(3.3)$ & $9(3.2)$ & $6(3.5)$ & \multirow{3}{*}{$p=0.9841$} \\
\hline B & $109(24.3)$ & $68(24.4)$ & $41(24.1)$ & \\
\hline $\mathrm{C}$ & $325(72.4)$ & $202(72.4)$ & $123(72.4)$ & \\
\hline \multicolumn{5}{|l|}{$\mathrm{AFP}, \mathrm{ng} / \mathrm{mL}$} \\
\hline Median (range) & $113.0(0.7-1148,415.7)$ & $94.0(0.7-1148,415.7)$ & $119.8(1-319,713.0)$ & \multirow{3}{*}{$p=0.5968^{*}$} \\
\hline$<400$ & $266(59.2)$ & $166(59.5)$ & $100(58.8)$ & \\
\hline$\geq 400$ & $172(38.3)$ & $103(36.9)$ & $69(40.6)$ & \\
\hline \multicolumn{5}{|l|}{ Immunotherapy treatment } \\
\hline Anti-PD(L)-1 monotherapy & $379(84.4)$ & $232(83.2)$ & $147(86.5)$ & \multirow{5}{*}{$p=0.1690$} \\
\hline Anti-PD(L)-1 + CTLA-4 combination & $28(6.2)$ & $18(6.5)$ & $10(5.9)$ & \\
\hline Anti-PD (L)-1 + TKI combination & $24(5.3)$ & $19(6.8)$ & $5(2.9)$ & \\
\hline Anti-PD (L)-1 + other combination & $16(3.6)$ & $10(3.6)$ & $6(3.5)$ & \\
\hline Anti-CTLA-4 monotherapy & $2(0.4)$ & - & $2(1.2)$ & \\
\hline
\end{tabular}

HCC, hepatocellular carcinoma; EIOP, early immunotherapy period; CTP, Child-Turcotte Pugh; BCLC, Barcelona clinic liver cancer; CTLA4, cytotoxic T-cell lymphocyte-associated antigen-4. * Estimates computed excluding missing data.

ment with ICI between 2017 and 2019 (online suppl. Table 1; see www.karger.com/doi/10.1159/000519108 for all online suppl. material). Patients' demographics and clinical data were collected retrospectively, curated, and updated at each participating center. Eligible patients had to fulfill the following inclusion criteria: (1) diagnosis of HCC made by histopathology or imaging criteria according to the American Association for the Study of Liver Disease
[14] and European Association for the Study of the Liver [15] guidelines; (2) be eligible for ICI monotherapy or combinations for HCC not amenable to curative or locoregional therapy following local multidisciplinary tumor board review; and (3) measurable disease according to RECIST 1.1 criteria [16] at ICI commencement. At the censoring date of 20th of February 2020, the multicenter database included 449 eligible patients.
Antibiotics and Greater Benefit from Immunotherapy in Liver Cancer 
Patients received ICI therapy either as monotherapy or combination therapy between 2017 and 2019, and this was continued until disease progression, unacceptable toxicity, or withdrawal of consent. Clinicopathologic variables (Table 1) were collated into a data collection proforma. Radiological disease staging by magnetic resonance imaging or computerized tomography was conducted prior to therapy commencement and at approximately 9 weekly intervals. Ethical approval to conduct this study was granted by the Imperial College Tissue Bank (reference number: R16008). The Institutional Review Board in each participating institution approved the study protocol. All study-related procedures and data collection were conducted in accordance with the Declaration of Helsinki and in accordance with Good Clinical Practice.

\section{Study Endpoints}

Clinical endpoints evaluated in association with ATB included OS, defined as the time from the initiation of ICI to patient death or loss to follow-up, PFS, defined as the time from the initiation of ICI to disease progression or death/loss to follow-up, the objective response rate (ORR) and the disease control rate (DCR) to ICI therapy, calculated on the basis of the best overall response achieved throughout the course of ICI therapy as assessed by the treating physician according to RECIST v1.1 criteria [16]. Patients were classified as achieving an objective response if a complete response (CR) or partial response (PR) was detected on restaging scans at 9-12 weeks intervals from ICI commencement.

\section{Evaluation of ATB Exposure}

In view of previous evidence showing a time-dependent association between ATB exposure and outcome from ICI, with greater influence of early ATB exposure, we categorized patients receiving ATB therapy within 30 days from starting ICI therapy (pATB) and considered them together with patients who were treated with ATB up to 30 days after cycle 1 of immunotherapy (eATB), defining this exposure window as the early immunotherapy period ([EIOP] i.e., -30 days to +30 days from ICI dosing). This was chosen as primary exposure measure according to the literature, which univocally suggests early ATB exposure as a detrimental factor influencing ICI efficacy [17].

To properly weigh our results based on potential confounders, baseline associations between EIOP ATB therapy grouping and each baseline characteristic were evaluated. Considering the possible interactions, large variations, and unbalanced distribution across patient's subgroups, fixed, preplanned multivariable regression models were used to weight each clinical outcome analysis according to the EIOP ATB therapy grouping [18, 19]. Covariates were chosen with a clinical prioritization and baseline covariates, which are known to be significantly associated to each other were excluded (e.g., Barcelona clinic liver cancer [BCLC] stage includes performance status and Child-Turcotte Pugh [CTP] liver function). Moreover, to choose appropriate covariates, mutual correlations were also formally evaluated prior to defining multivariable models (data not shown).

The included relevant variables were gender [20], age ( $<70$ vs. $>70$ years) [21], viral etiology (yes vs. no), CTP class (A vs. B), BCLC stage (A vs. B/C), ICI therapy (PD-[L] 1 monotherapy vs. others, including combinations), treatment line (first vs. nonfirst line), and AFP ( $\geq 400$ vs. $<400$ ). An additional analysis of OS and PFS using EIOP clustered as beta-lactams, quinolones, other single agent ATB, and ATB combinations was also performed.
Last, we took into consideration the lead-in time bias caused by the time-dependent nature of eATB exposure. As patients in the eATB group could had been treated with ATB up to 30 days after treatment commencement, we had to assume that potentially their immunotherapy exposure lasted at least 30 days, while is presumable that early progressors (e.g., patients who discontinued the treatment within the first 30 days) were included among the noneATB patients only. Therefore, in order to overcome that bias, a further clinical outcomes analysis was performed after a 30-day landmark selection, including only patients with a minimum treatment duration of 30 days. We further evaluated whether EIOP ATB therapy could stratify patient's clinical outcomes in the prespecified restricted group of patients treated with PD1/PD-L1 checkpoint inhibitors monotherapy and those with CTP class A.

\section{Statistical Analysis}

Demographic data were summarized using descriptive statistics. The median OS and PFS were calculated using the univariate Kaplan-Meier survival method. The median period of follow-up was calculated according to the reverse Kaplan-Meier method. Log-rank tests were performed to compare OS and PFS according to ATB category. The $\chi^{2}$ test was used to compare categorical variables between the 2 cohorts and for the univariable analyses of ORR/DCR. Logistic regression was used for the multivariate analysis of ORR/DCR and to compute odds ratios with 95\% confidence intervals (CIs). Cox proportional hazards regression was used for the multivariate analysis of PFS and OS and to compute all the hazard ratios (HRs) for treatment discontinuation and death with $95 \%$ CIs. The alpha level for all analyses was set to $p<0.05$. All statistical analyses were performed using MedCalc Statistical Software version 19.3.1 (MedCalc Software Ltd., Ostend, Belgium; https://www.medcalc.org; 2020).

\section{Results}

\section{Baseline Cohort Characteristics}

In this multinational retrospective study, clinical data from 449 patients with HCC treated with ICI from 12 centers in the USA $(n=250,55 \%)$, Europe $(n=109,26 \%)$, and Asia $(n=91,20 \%)$ were collated and reviewed, after exclusion of 15 patients classified as Child-Pugh class C, ECOG performance status of 3 (i.e., BCLC stage D). ATB were administered within the EIOP time window in 170 patients (37.9\%). Within the EIOP (EIOP+) category, 82 patients received ATB prior to ICI (pATB, 18.3\%) and 128 patients within 30 days of ICI initiation (eATB, $28.5 \%$ ), with 40 of them having received ATB in both pATB and eATB time windows. Empirical treatment, that is, fever raising the possibility of an infection of unclear source, was the most common indication for all those who received pATB (59.8\%) and eATB (42.9\%). The most common types of ATB prescribed were betalactams and quinolones in both the pATB $(41.4 \%$ and $34.1 \%$, respectively) and the eATB groups $(67.9 \%$ and 


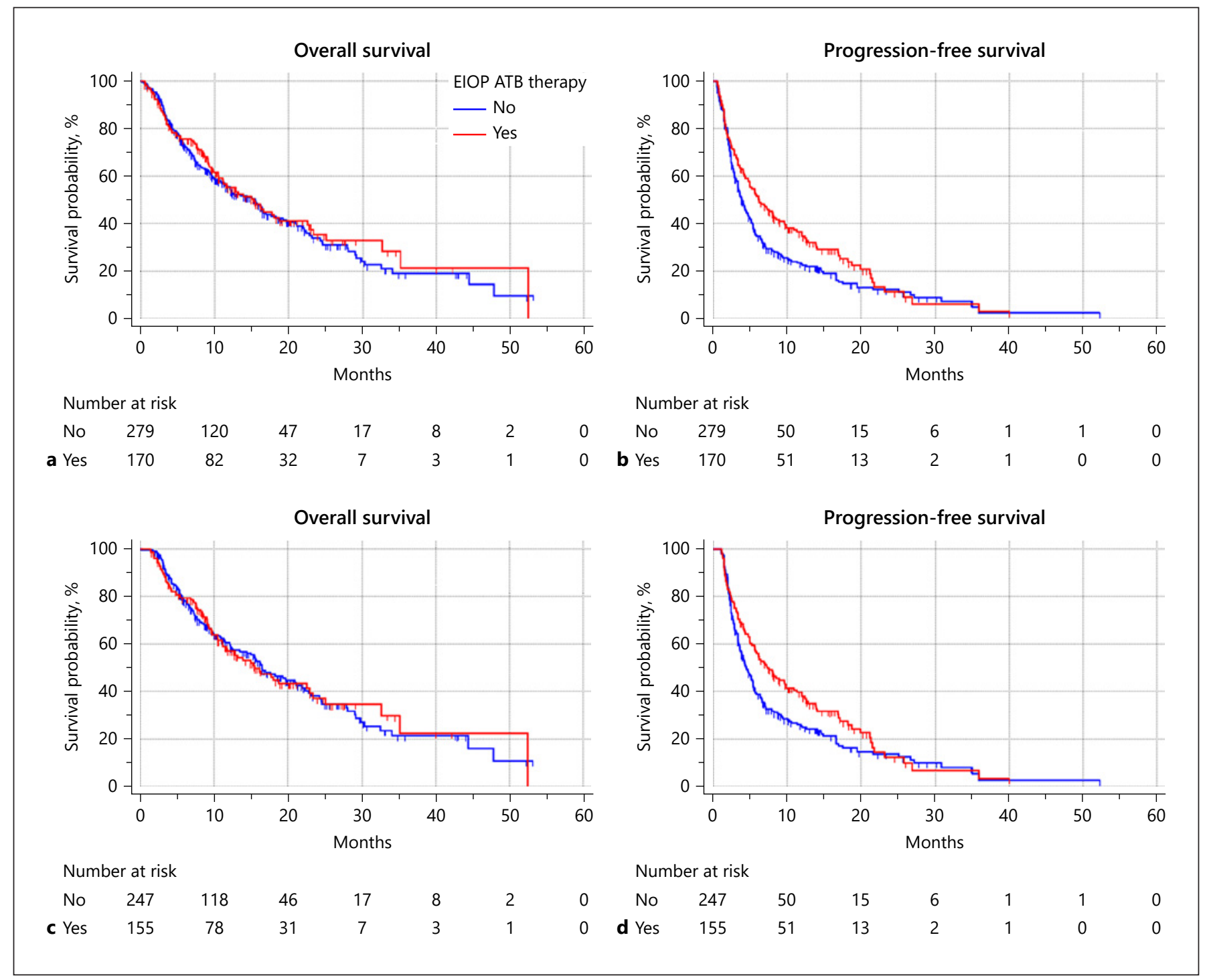

Fig. 1. The influence of early ATB exposure (EIOP) on OS (a) and TTF (b) in the overall patient population $(n=450)$ and in the 4 -week landmark time survival analysis population $(n=402)$ (c, d). EIOP, early immunotherapy period; ATB, antibiotics; OS, overall survival; TTF, time to treatment failure.

$35.1 \%$, respectively). Detailed indications and ATB types are reported in online suppl. Tables 2 and 3. Most patients received PD-1/PD-L1 inhibitor single-agent therapy $(n=$ $379,84.4 \%)$. At ICI initiation, most patients were of BCLC stage C $(n=325,72.4 \%)$, with a Child-Pugh class of A $(n=332,73.9 \%)$. Table 1 summarized all key baseline patients' characteristics for the overall study population and according to EIOP grouping. With the exception of the proportion of patients who underwent to previous surgical resection $(p=0.0079)$, none among the clinical characteristics resulted to be significantly associated to cohort grouping according to the EIOP status. After a median follow-up of 17.0 months (95\% CI: 15.5-53.2), the median OS and PFS of the entire cohort were 15.4 months (95\% CI: 12.6-16.6; 241 events) and 4.4 months (95\% CI: $3.7-5.3 ; 344$ events), respectively. Among the 428 evaluable patients for radiologic disease response, 26 (6.1\%) achieved a CR and 50 (11.7\%) a PR (11.7\%), leading to an ORR of $17.8 \%$ (95\% CI: 13.9-22.2). Stable disease was reported for 174 patients (40.7\%), resulting in a DCR of 58.4\% (95\% CI: 51.4-66.1). Patients who experienced a $\mathrm{PR} / \mathrm{CR}$ to ICI achieved a significantly longer median OS 
Table 2. Multivariable analyses illustrating the relationship between early ATB exposure (EIOP) and key efficacy outcomes in patients with HCC treated with immunotherapy

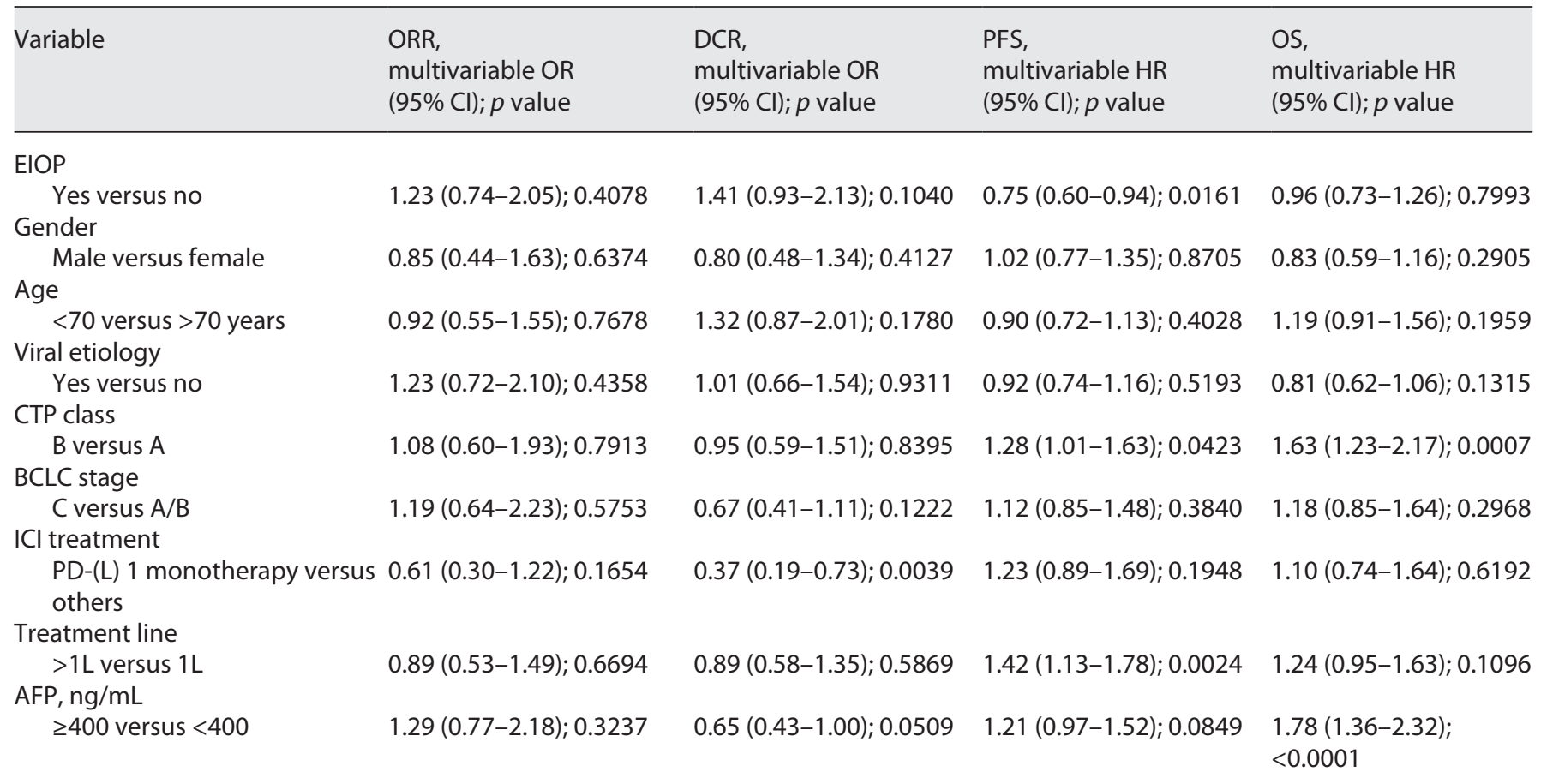

ORR and DCR analyses: 417 patients included. PFS and OS analyses: 438 patients included. ICl, immune checkpoint inhibitor; HCC, hepatocellular carcinoma; ATB, antibiotics; OS, overall survival; PFS, progression-free survival; ORR, objective response rate; $D C R$, disease control rate; EIOP, early immunotherapy period; CTP, Child-Turcotte Pugh; BCLC, Barcelona clinic liver cancer; OR, odds ratio; Cl, confidence interval; $\mathrm{HR}$, hazard ratio.

(not reached; 76 events) than patients who experienced a stable/progressive disease (12.1 months; 95\% CI: 9.8$15.4 ; 214$ events) (HR 0.37, 95\% CI: $0.27-0.51 ; p<0.0001$ ) (online suppl. Fig. 1).

\section{The Relationship between EIOP ATB Therapy and} Efficacy Outcomes from ICI

Despite a concordant trend, EIOP exposure was neither associated to the ORR (20.2\% [95\% CI: 14.0-28.2] for the EIOP+ group and $16.1 \%$ [95\% CI: 11-6-21.8] for EIOP-; $p=0.2808)$, nor to the DCR (63.1\% [95\% CI: 51.676.3] for EIOP+ and 55.4\% [95\% CI: 46.7-65.4] for EIOP$; p=0.1144)$. In the EIOP+ group, the median OS was 15.3 months (95\% CI: 11.1-52.5; 88 events), compared to 15.4 months (95\% CI: 11.3-17.6; 153 events) for EIOP- patients (log-rank: $p=0.6275$; HR 0.93 [95\% CI: 0.72-1.21], Fig. 1a), while the median PFS was 6.1 months (95\% CI: 4.6-8.1; 124 events) for EIOP+ patients and 3.7 months (95\% CI: 3.2-4.5; 220 events) for EIOP- ones (log-rank: $p=0.0135$; HR 0.74 [95\% CI: 0.60-0.93], Fig. 1b). Multi- variable analyses of ORR, DCR, OS, and PFS are illustrated in Table 2 and confirm the relationship between ATB EIOP exposure and longer PFS (HR 0.75 [95\% CI: 0.60-0.94]; $p=$ $0.0161)$. Online suppl. Table 4 reports the univariable analysis of PFS and OS according to the EIOP ATB class. When clustered as beta-lactams, quinolones, others single agent ATB, and ATB combinations, a significantly prolonged PFS was reported for patients on EIOP quinolones only (HR $0.56,95 \%$ CI: $0.34-0.91 ; p=0.0217$ ), while no effects on OS were found. At the multivariable, no effect on PFS and OS was confirmed (data not shown).

\section{Thirty Days Landmark Analysis}

After the 30-day landmark selection, 402 patients were included in the analysis. Among them, 155 (38.6\%) were grouped as EIOP+ and 247 (61.4\%) and EIOP-. EIOP exposure was neither associated to the ORR $(22.1 \%$ [95\% CI: 15.3-30.9] for EIOP+ and 17.3\% [95\% CI: 12.4-23.4] for EIOP-; $p=0.2416)$, nor to the DCR (66.9\% [95\% CI: 54.681.1] for EIOP+ and 59.1\% [95\% CI: 49.7-69.7] for EIOP-; 
$p=0.1202)$. In the EIOP- group, the median OS was 15.4 months (95\% CI: 11.6-52.4; 78 events), compared to 16.4 months (95\% CI: 13.4-21.9; 127 events) of the EIOPgroup (log-rank: $p=0.9812$; HR 1.01 [95\% CI: 0.75-1.33], Fig. 1c), while the median PFS was 7.2 months (95\% CI: 5.5-9.8; 110 events) for the EIOP+ group, and 4.4 months (95\% CI: 3.7-5.3; 192 events) for the EIOP- group (logrank: $p=0.0171$; HR 0.74 [95\% CI: 0.59-0.94], Fig. 1d). Multivariable analyses confirmed the relationship between ATB EIOP exposure and longer PFS (HR 0.73 [95\% CI: 0.57-0.93]; $p=0.0130$, online suppl. Table 5).

\section{Child-Pugh Class A and PD-1/PD-L1 Inhibitors}

Monotherapy Ancillary Analysis

Table 3 provides a summary of the Child-Pugh class A and the PD-1/PD-L1 inhibitors monotherapy ancillary analyses. Among the PD-1/PD-L1 monotherapy restricted group, EIOP ATB therapy showed no significant association with OS ( $p=0.5462)$ (online suppl. Fig. 2a) and ORR ( $p=0.3378)$, while was significantly associated with a longer PFS (HR $=0.75$ [95\% CI: 0.60-0.95]; $p=0.0205$ ) (online suppl. Fig. 2b) and a higher DCR (61.4\% vs. 50.9\%, $p=0.0494)$. Among the CTP class A restricted group, EIOP ATB therapy showed no association with OS ( $p=$ $0.9652)$ (online suppl. Fig. 2c), DCR $(p=1,476)$ and ORR ( $p=0.2696)$, while resulted to be significantly associated with a prolonged PFS (HR $=0.76$ [95\% CI: 0.58-0.98]; $p=0.0362$ ) (online suppl. Fig. 2d).

\section{Discussion}

Drug-drug interactions are at the focus of intense scrutiny in the context of ICI therapy, given their potential to blunt immune responsiveness and affect oncological outcomes [22]. The homeostatic equilibrium of the gut microbiome has become an increasingly recognized determinant of response to ICI, in view of the bidirectional cross talk between the diverse commensal species residing in the intestinal lumen and the host immunity [23]. ATB therapy can induce protracted changes to the gut microbiome and has emerged as a tumor-agnostic determinant of response and survival to ICI across a growing number of malignancies [24].

This is the first study to report on the effect of ATB on key oncological outcomes following immunotherapy for HCC, a disease where alterations of the gut-liver axis are an important pathogenic determinant [25]. It has been established that dysfunction of the intestinal barrier permits increased translocation of intestinal bacteria in 
chronic liver disease [26]. Continued exposure to lipopolysaccharide from gut bacteria is an important contributor to liver cirrhosis and cancer development via activation of the TGF- $\beta$ pathway, a key molecular driver of proliferative and antiapoptotic signals in hepatocytes [27, 28 ]. Once HCC is established, the gut-liver axis continues to influence the antitumor immune response and perturbation of the gut microbiome, in which ATB can have a direct effect on the HCC microenvironment [29].

Our study portrays a direction and strength of association between ATB and outcome from immunotherapy in HCC that is in deep contrast to other oncological indications. While ATB therapy was not associated with OS in our study, we found that EIOP dosing preludes to a significant delay in disease progression or death following ICI therapy, as measured by PFS. Interestingly, early ATB exposure was not associated with characteristics of severity of chronic liver disease, performance status, or HCC stage and persisted as an independent predictor of PFS in multivariable models adjusted for these variables. This provides further credence to a true pathophysiologic rather than merely associative link between ATB and improved disease control from ICI therapy, a finding that places this study at odds with most studies conducted on this topic in other cancer types [24].

The universally detrimental effect on outcomes observed for prior and early but not concurrent ATB use across malignancies is thought to relate to the negative effect of ATB in reducing diversity and taxonomy in the commensal bacteria, producing a reduction in Bifidobacteria, Akkermansia, and Ruminococcus, while favoring growth of other particular bacterial species in the gut microbiota such as Bacteroides [30, 31]. Such bacteria are postulated to induce immunosuppression through promotion of myeloid-derived suppressor cells, FOXP3+ and $\mathrm{CD} 4+\mathrm{CD} 25+\mathrm{T}$-regulatory cells, and production of systemic prostaglandins that are negatively associated with ICI response [24]. Perturbation of the microbiota as a mechanism linking ATB treatment and response to immunotherapy is beginning to be confirmed through prospective testing. A recent study has demonstrated the ability of immunostimulatory commensal bacteria or fecal transplantation from ICI-responding renal cell carcinoma patients to rescue ICI-resistant, renal cell carcinoma-bearing mice [32]. In immunotherapy-resistant melanoma, fecal microbiota transplantation has been shown to rescue immunotherapy resistance in patients [23].

HCC is set apart from other solid tumors in that it develops in the context of cirrhosis, a pathological state already associated with an immunosuppressive microbi- ome [33]. Cirrhosis is accompanied by gut microbial dysbiosis with evidence of decreased beneficial bacteria and increased immunosuppressive bacteria. These findings have been corroborated many times in studies with mouse models finding reduced Bifidobacterium and increased gram-negative bacteria, such as Bacteroides and Escherichia Coli, speculated to contribute to HCC progression [34]. It is therefore plausible that disruption of this immunosuppressive interaction by ATB may favor response to ICI. A candidate mechanism recently demonstrated in HCC and non-HCC mice is that primary bile acids in the gut promote expression of CXCL16 and NKT cell recruitment in the liver [29]. Eradication of bacteria that metabolize primary bile acids by ATB in these mice enhanced antitumor immunity and reduced tumor growth.

Considering the wider tumor microenvironment, unlike lung, bladder cancer, and melanoma, liver cancer is characterized by a uniquely immunosuppressive milieu at baseline [35], dominated - amongst other mechanisms by abundant recruitment of myeloid suppressor cells and tumor-associated macrophages, which directly inhibit cytotoxic T cells and produce chemokines including CCL17, CCL18, and CCL22, which further attract Tregs [36, 37]. The effect of ATB on such a wide spectrum of immunosuppressive innate immune cells is mostly unknown.

The above factors may account for a different relationship between ATB exposure and ICI. Although our study lacks correlative data from stool samples that would enable us to characterize the microbiome changes associated with ATB administration, it lends clinical support to the idea that HCC develops in the setting of a unique interaction between the gut microbiome and the immune system.

There are a number of reasons why ATB might have exerted a differential impact on PFS as opposed to OS. PFS is more directly reflective of immunotherapy-induced therapeutic benefit as opposed to OS, a measure that, in heavily pretreated patients who were particularly heterogeneous in terms of treatment lines, might have been more predominantly influenced by post-progression therapy. As shown in Table 1, nearly half of the patients were treated with immunotherapy after 1 prior line of systemic treatment. Besides heterogeneity in therapy, OS is deeply influenced by liver functional reserve in advanced HCC patients. It is therefore possible that mortality due to liver decompensation and worsening of underlying chronic liver disease might have masked a measurable effect of ATB on OS.

The current first line choice for immunotherapy in HCC is the combination of atezolizumab and bevacizum$\mathrm{ab}$ [6]. Our study provides no primary data to support our 
conclusion in this subtype of therapy. The majority of patients treated in our study were in fact treated with anti PD-1/PD-L1 monotherapy. While we hypothesize that the effect is likely to be preserved, given the synergistic effect of anti-PD-L1 and anti-VEGF therapy on enhancing antitumor immunity, further studies should prove whether the association between ATB and outcome from PD-1/VEGF combinations holds true in adequately powered prospective clinical studies.

Overall, our study reports on an international, multicenter, and geographically heterogeneous study with a sample size relatively larger than the studies conducted in other cancers. Our study is limited by its retrospective nature and by the lack of molecular analyses on the stool microbiota, a point that should be addressed in prospective studies. Additionally, given the proposed mechanisms relating to disruption of the gut microbiota, it would be ideal for independent host factors known to influence the gut ecosystem, such as diet, alcohol, and smoking, to be thoroughly accounted for. In conclusion, our findings demonstrate a significant difference in duration of therapeutic benefit in patients with advanced HCC treated with ICI receiving ATB in the early period prior to or after ICI initiation. This relationship cannot be explained by differences in baseline liver function, functional status, or immunotherapy regimen administered between patient groups. Although the delay of progression observed in our study does not translate to enhanced OS, the results stand as valuable initial evidence to clearly warrant this as an area of further investigation, especially in light of ongoing trials in other solid tumors investigating the modulation of the microbiome as a strategy to potentiate the efficacy of immunotherapy and reverse immunotherapy resistance [38].

\section{Acknowledgements}

The authors would like to acknowledge the infrastructure support provided by Imperial Experimental Cancer Medicine Center, Cancer Research UK Imperial Center, the Imperial College Healthcare NHS Trust Tissue Bank, and the Imperial College BRC. The authors are grateful to Thoetchai Peeraphatdit (University of Chicago, Chicago, IL, USA) and ChiehJu Lee (Taipei Veterans Hospital, Taiwan) for their help with clinical data collection.

\section{Statement of Ethics}

This project received authorization for the retrospective use of patient data from the Imperial College Tissue Bank (reference number: R16008). The study was conducted in accordance with the principles stipulated in the Declaration of Helsinki and following Good Clinical Practice standards. The date of ethical approval was on March 20, 2018. Written consent was waived due to the retrospective nature of the study.

\section{Conflict of Interest Statement}

M.K. is the editor in chief of Liver Cancer. N.N. is an editorial board member of Liver Cancer. D.J.P. received lecture fees from ViiV Healthcare, Roche, EISAI, Bayer Healthcare, Falk Foundation; travel expenses from BMS, MSD, Roche, and Bayer Healthcare; consulting fees from Mina Therapeutics, EISAI, Roche, AstraZeneca, H3B, DaVolterra; received research funding (to institution) from MSD and BMS. DB has received lecture and speaker fees from Bayer Healthcare and the Falk Foundation Germany. Y.H.H. has received advisory board/ consulting fees for BMS, MSD, Bayer Healthcare, IPSEN, EISAI, Gilead, and Lilly. A.S. received research funding (to institution) from AstraZeneca, Exelixis, BMS, and Clovis; advisory board/consulting fees from BMS, AstraZeneca, and Exelixis. A.C. received lecture fees from MSD, AstraZeneca, Astellas, and Novartis; consulting fees from MSD, Roche, AstraZeneca, Novartis, and BMS. There are no other personal or financial conflicts of interest to disclose. T.P. reports receiving institutional research funding from Lilly. N.P. reports receiving consulting fees from Amgen, Merck Serono, Servier; lectures fees from AbbVie, Gilead, Lilly; travel expenses from Amgen, ArQule; and institutional research funding from Basilea, Merck Serono, Servier. L.R. reports receiving consulting fees from Amgen, ArQule, AstraZeneca, Basilea, Bayer, Celgene, Eisai, Exelixis, Genenta, Hengrui, Incyte, Ipsen, Lilly, MSD, Nerviano Medical Sciences, Roche, Sanofi; lectures fees from AbbVie, Amgen, Eisai, Gilead, Incyte, Ipsen, Lilly, Roche, Sanofi; travel expenses from Ipsen; and institutional research funding from Agios, ARMO BioSciences, AstraZeneca, BeiGene, Eisai, Exelixis, Fibrogen, Incyte, Ipsen, Lilly, MSD, Nerviano Medical Sciences, Roche, and Zymeworks.

\section{Funding Sources}

D.J.P. is supported by grant funding from the Wellcome Trust Strategic Fund (PS3416) and has received direct project funding by the NIHR Imperial Biomedical Research Center and ITMAT Push for Impact Grant Scheme 2019. The views expressed are those of the authors and not necessarily those of the NIHR or the Department of Health and Social Care. D.B. is supported by the BertaOttenstein Programme, Faculty of Medicine, University of Freiburg.

\section{Author Contributions}

D.J.P., M.N., P.F., and A.C contributed to the study concept and design. All authors contributed to the acquisition of data and critical revision of the manuscript for important intellectual con- 
tent. P.F., M.N., and A.C analyzed and interpreted the data. M.N., P.F., and A.C drafted the manuscript. P.F., D.J.P., and A.C. contributed to statistical analysis. D.J.P obtained funding. D.J.P. and A.C supervised the study.

\section{Data Availability Statement}

The data that support the findings are available after reasonable request.

\section{References}

1 Rawla P, Sunkara T, Muralidharan P, Raj JP. Update in global trends and aetiology of hepatocellular carcinoma. Contemp Oncol. 2018;22:141-50.

2 Colagrande S, Inghilesi AL, Aburas S, Taliani GG, Nardi C, Marra F. Challenges of advanced hepatocellular carcinoma. World J Gastroenterol. 2016;22:7645-59.

3 Lohitesh K, Chowdhury R, Mukherjee S. Resistance a major hindrance to chemotherapy in hepatocellular carcinoma: an insight. Cancer Cell Int. 2018;18:44.

4 Galluzzi L, Chan TA, Kroemer G, Wolchok JD, López-Soto A. The hallmarks of successful anticancer immunotherapy. Sci Transl Med. 2018;10(459):eaat7807.

5 Pinato DJ, Guerra N, Fessas P, Murphy R, Mineo T, Mauri FA, et al. Immune-based therapies for hepatocellular carcinoma. Oncogene. 2020;39:3620-37.

6 Finn RS, Qin S, Ikeda M, Galle PR, Ducreux M, Kim TY, et al. Atezolizumab plus bevacizumab in unresectable hepatocellular carcinoma. N Engl J Med. 2020;382:1894-905.

7 Iglesias-Santamaria A. Impact of antibiotic use and other concomitant medications on the efficacy of immune checkpoint inhibitors in patients with advanced cancer. Clin Transl Oncol. 2020;22:1481-90.

8 Becattini S, Taur Y, Pamer EG. Antibiotic-induced changes in the intestinal microbiota and disease. Trends Mol Med. 2016;22:458-78.

9 Huang XZ, Gao P, Song YX, Xu Y, Sun JX, Chen XW, et al. Antibiotic use and the efficacy of immune checkpoint inhibitors in cancer patients: a pooled analysis of 2,740 cancer patients. Oncoimmunology. 2019; 8: e1665973.

10 Pierrard J, Seront E. Impact of the gut microbiome on immune checkpoint inhibitor efficacy: a systematic review. Curr Oncol. 2019; 26:395-403.

11 Pinato DJ, Howlett S, Ottaviani D, Urus $\mathrm{H}$, Patel A, Mineo T, et al. Association of prior antibiotic treatment with survival and response to immune checkpoint inhibitor therapy in patients with cancer. JAMA Oncol. 2019;5:1774-8.

12 Cortellini A, Tucci M, Adamo V, Stucci LS, Russo A, Tanda ET, et al. Integrated analysis of concomitant medications and oncological outcomes from PD-1/PD-L1 checkpoint inhibitors in clinical practice. J Immunother Cancer. 2020;8:2020.

13 Yu LX, Schwabe RF. The gut microbiome and liver cancer: mechanisms and clinical translation. Nat Rev Gastroenterol Hepatol. 2017;14: $527-39$.
14 Heimbach JK, Kulik LM, Finn RS, Sirlin CB, Abecassis MM, Roberts LR, et al. AASLD guidelines for the treatment of hepatocellular carcinoma. Hepatology. 2018;67:358-80.

15 European Association for the Study of the Liver. Electronic address eee, European association for the study of the L: EASL clinical practice guidelines: management of hepatocellular carcinoma. J Hepatol. 2018;69:182236.

16 Eisenhauer EA, Therasse P, Bogaerts J, Schwartz LH, Sargent D, Ford R, et al. New response evaluation criteria in solid tumours: revised RECIST guideline (version 1.1). Eur J Cancer. 2009;45:228-47.

17 Pinato DJ, Gramenitskaya D, Altmann DM, Boyton RJ, Mullish BH, Marchesi JR, et al. Antibiotic therapy and outcome from immune-checkpoint inhibitors. J Immunother Cancer. 2019;7:287.

18 Thompson FT. Examples of easily explainable suppressor variables in multiple regression research. Mult Lin Reg View. 1997.

19 Woolley KK. How variables uncorrelated with the dependent variable can actually make excellent predictors: the important suppressor variable case; 1997.

20 Valodara AM, Sr KJ. Sexual dimorphism in drug metabolism and pharmacokinetics. Curr Drug Metab. 2019;20:1154-66.

21 Shin J, Yu JH, Jin YJ, Suh YJ, Kim DH, Byun $S$, et al. Effective therapeutic options for elderly patients with hepatocellular carcinoma: a nationwide cohort study. Medicine. 2019; 98:e16150.

22 Hussain N, Naeem M, Pinato DJ. Concomitant medications and immune checkpoint inhibitor therapy for cancer: causation or association? Hum Vaccin Immunother. 2021; 17(1):55.

23 Baruch EN, Youngster I, Ben-Betzalel G, Ortenberg R, Lahat A, Katz L, et al. Fecal microbiota transplant promotes response in immunotherapy-refractory melanoma patients. Science. 2020;371(6529):602.

24 Spakowicz D, Hoyd R, Muniak M, Husain M, Bassett JS, Wang L, et al. Inferring the role of the microbiome on survival in patients treated with immune checkpoint inhibitors: causal modeling, timing, and classes of concomitant medications. BMC Cancer. 2020;20:3832020.

25 Albillos A, de Gottardi A, Rescigno M. The gut-liver axis in liver disease: pathophysiological basis for therapy. J Hepatol. 2020;72:55877.

26 Lin RS, Lee FY, Lee SD, Tsai YT, Lin HC, Lu $\mathrm{RH}$, et al. Endotoxemia in patients with chronic liver diseases: relationship to severity of liver diseases, presence of esophageal varices, and hyperdynamic circulation. J Hepatol. 1995;22:165-72.

27 Seki E, De Minicis S, Osterreicher CH, Kluwe J, Osawa Y, Brenner DA, et al. TLR4 enhances TGF-beta signaling and hepatic fibrosis. Nat Med. 2007;13:1324-32.

28 Dapito DH, Mencin A, Gwak GY, Pradere JP Jang MK, Mederacke I, et al. Promotion of hepatocellular carcinoma by the intestinal microbiota and TLR4. Cancer cell. 2012;21:50416.

29 Ma C, Han M, Heinrich B, Fu Q, Zhang Q, Sandhu M, et al. Gut microbiome-mediated bile acid metabolism regulates liver cancer via NKT cells. Science. 2018;360:2018.

30 Gopalakrishnan V, Spencer CN, Nezi L, Reuben A, Andrews MC, Karpinets TV, et al. Gut microbiome modulates response to antiPD-1 immunotherapy in melanoma patients. Science. 2018;359:97-103.

31 Zheng Y, Wang T, Tu X, Huang Y, Zhang H, Tan D, et al. Gut microbiome affects the response to anti-PD-1 immunotherapy in patients with hepatocellular carcinoma. J Immunother Cancer. 2019;7:193.

32 Derosa L, Routy B, Fidelle M, Iebba V, Alla L, Pasolli E, et al. Gut bacteria composition drives primary resistance to cancer immunotherapy in renal cell carcinoma patients. Eur Urol. 2020;78:195-206.

33 Herbst DA, Reddy KR. Risk factors for hepatocellular carcinoma. Clin Liver Dis. 2012;1: $180-2$.

34 Ponziani FR, Nicoletti A, Gasbarrini A, Pompili M. Diagnostic and therapeutic potential of the gut microbiota in patients with early hepatocellular carcinoma. Ther Adv Med Oncol. 2019;11:1758835919848184.

35 Levitsky J. Does the liver provide immunosuppressive advantage? Clin Liver Dis. 2019; 13:180-3.

36 Lu C, Rong D, Zhang B, Zheng W, Wang X, Chen Z, et al. Current perspectives on the immunosuppressive tumor microenvironment in hepatocellular carcinoma: challenges and opportunities. Mol Cancer. 2019;18:130.

37 Salmaninejad A, Valilou SF, Soltani A, Ahmadi S, Abarghan YJ, Rosengren RJ, et al. Tumor-associated macrophages: role in cancer development and therapeutic implications. Cell Oncol. 2019;42:591-608.

38 Daillere R, Derosa L, Bonvalet M, Segata N, Routy B, Gariboldi M, et al. Trial watch: the gut microbiota as a tool to boost the clinical efficacy of anticancer immunotherapy. Oncoimmunology. 2020;9:1774298. 